\title{
Presidential Address 2014
}

\section{Moving towards an era free of HIV}

\section{Himali Perera ${ }^{1}$}

Our Chief Guest today,

Professor Ahtula Kaluarachchi,

Guest of Honour, Dr Charlotte Bell

Past Presidents

Members of the council,

Fellows of the College,

Members of the College,

Distinguish invitees,

Ladies and gentlemen,

It is my great pleasure addressing you at the inauguration of the $19^{\text {th }}$ Scientific Sessions of the Sri Lanka College of Venereologists.

"Moving towards an era free of HIV" is the theme of our sessions this year. This theme was based on the global vision to have zero new infections of HIV by year 2030 .

To begin with, I would like to draw your attention towards the global and regional situation of HIV.

\section{Global situation}

Globally, new HIV infection rate has fallen by nearly $33 \%$ during the past 10 years, with a $40 \%$ drop in sub-Saharan Africa and a 50\% drop in the Caribbean. More than thirteen million people were on HIV treatment globally by the end of 2013. More HIV patients are now surviving due to effective treatment.

\section{Situation in South East Asia}

During 2011, the South East Asian region had estimated 211,000 people newly infected with HIV. This is a $35 \%$ reduction compared to 2001 . The HIV epidemic is in decline in the South East Asian Region

'President, Sri Lanka College of Venereologists

The Sri Lanka Journal of Venereology Vol. 5, No. 1, December 2014 and has stabilized in many countries. Four high burden countries, India, Myanmar, Nepal and Thailand have reduced new HIV infection by more than $50 \%$ since 2000. In contrast, Bangladesh, Bhutan Indonesia and SriLanka have experienced an increase of $25 \%$ since 2000. Overall with improved availability and accessibility to antiretroviral treatment disease specific death rate has fallen by $14 \%$ since 2005 , in the region.

\section{Situation in Sri Lanka}

Over the past three decades, Sri Lanka has remained as low prevalence country. The first Sri Lankan who acquired the infection in a foreign country was reported in1987. The first Sri Lankan to acquire the infection locally was diagnosed in 1989. By end of 2013, 1995 HIV positive patients have been reported to the National STD/AIDS Control Programme (NSACP). A total number of 320 deaths and 71 cases of mother to child transmission (MTCT) have been reported up to end of June 2014. HIV screening was done on 586,760 by end of 2013, out of which 196 were confirmed positive.

Over the years, there has been a trend of diagnosing HIV patients at the stage of AIDS, which carry a high mortality. Among reported cases, approximately $25 \%$ were in the stage of AIDS. This can be minimised by increasing awareness among people to seek HIV testing following high risk behaviours and improving HIV testing and counselling services in the country.

In $2013,48 \%$ of new HIV cases were reported among heterosexuals followed by bisexuals amounting to $27 \%$. MTCT was responsible $6 \%$ of cases. Intravenous drug use and sharing of needle/syringes contributed only to $2 \%$ of cases.During 2013 , the 
highest number of HIV cases were reported from the age group of 25 to 49 years. Colombo and Gampaha Districts had the highest rates of reported cases, while Monaragala, Matara and Kilinochchi districts reported the lowest HIV rates.

In Sri Lanka the epidemic is propagated by most at risk groups such as men who have sex with men (MSM), female sex workers and intravenous drug uses. According to mapping of female sex workers in 2013, majority were from the Western province $(52 \%)$, followed by $11 \%$ in the Central Province.MSM were identified from all the provinces with variable numbers. The majority were fromWestern Province, followed by Southern Province and Central Province. The total number of drug users in the country was around 17,000. The majority of drug users were from the Western Province followed by North Western, Southern and Central Provinces.Beach boys are considered to be population at risk in Sri Lanka. Out of 873 estimated beach boys, $25 \%$ were from Ampara District, followed by $20 \%$ from Galle Districtt and 17\% from Kalutara District.

\section{Strategy towards an era free of HIV}

The strategy adopted towards achieving an era free of HIV in Sri Lanka consists of 3 main activities. This includes prevention of HIV transmission, early diagnosis and treatment, adherence and retention in care.

\section{Early Diagnosis}

Early diagnosis is vital in preventing HIV transmission. The National Reference Laboratory for HIV diagnosis is situated at the headquarters of the NSACP. In addition, each peripheral STD clinic has its own laboratory. The National Reference Laboratory of the NSACP provides services to the Central STD Clinic Colombo and functions as the reference laboratory to all peripheral STD laboratories and the private sector laboratories in the country. The National Reference Laboratory also provides viral load assay and CD4 count assay for HIV patients. Early neonatal HIV diagnosis in babies born to HIV infected mothers using DNA PCR was added to the routine diagnostic services since 2013. To further improve the testing capabilities for HIV, ELISA machines were provided to STD clinics in Chilaw, Kalutara, Hambanthota and Polonnaruwa. In 2013, a decision was made to introduce HIV testing to the routine antenatal screening package.

\section{Treatment}

Free of charge treatment for HIV is available in SriLanka since year 2004. There are 12 anti retroviral treatment (ART) centers in the country out of which seven centers are full time.

\section{Treatment cascade}

According to2013 data, out of estimated $2900 \mathrm{HIV}$ infected, only 1845 has been ever diagnosed, whichis around $64 \%$. Out of this, a total of 1236 were enrolled for HIV care. Since the beginning of the ART programme in 2004, 678 patients have been started on ART and $519(77 \%)$ of this were alive and on ART by the end of 2013. 14\% were dead and 9\% were lost to follow up.

Out of the patients started on ART, majority of persons living with HIV (PLHIV) are managed at central STD Clinic Colombo, though patients are from many parts of the country. Facilities at peripheral STD clinics are currently underutilized.

\section{Retention in care}

It is important to retain patients who are started on ART to reduce transmission of HIV and drug resistance. According to cohort analysis of patients on ART, it appears that we have done well in this regard.

\section{HIV-TB co infection}

With emergence of HIV epidemic tuberculosis has become a challenge. There are 19 cases of HIV/TB identified in Sri Lanka up to 2012.Therefore, TB screening in all HIV patients is mandatory. In addition, when there is a co infection, treatment of tuberculosis 
should be initiated and followed by ART to prevent immune reconstitution inflammatory syndrome.

\section{Prevention of HIV}

Targeted interventions at prevention of STI/HIV in key populations and vulnerable populations are the main key to reduce transmission of HIV in Sri Lanka. More than half of the reported HIV positive cases in Sri Lanka are migrant workers.Prisons provide a high risk environment for HIV transmission due to drug abuse, unsafe sexual practices, overcrowding, inadequate nutrition and poor health services. People employed in the entertainment and hospitality trade for tourists have access to risky sexual behaviors. In addition, tailor-made preventive messages to the general population including youth and women will help in prevention by improved awareness. Elimination of MTCT and prevention HIV transmission in health care settings could be achieved by specific targeted interventions.

\section{Sources of funding for national response $\mathbf{2 0 1 3}$}

Funding for national response is mainly (53.8\%) provided by the Sri Lankan government. This is utilized mainly for administrative purposes. The rest come from various donor agencies mainly for preventive activities.

\section{Treatment as a prevention strategy}

Some countries like USA,Brazil and Thailand have implemented treatment as a prevention strategy. To achieve treatment as a prevention RECRUIT - TEST - TREAT - RETAIN activities should be linked and be continuous.

\section{Evolution of ART criteria}

Criteria to start ART have evolved over the years. Initially it was started when CD4 count was below 200. At present ART is started at a CD4 count less than 500. Presently in Sri Lanka, ART is started regardless of $\mathrm{CD} 4$ count in sero-discordant couples, pregnant women "options $\mathrm{B} / \mathrm{B}+$ " " with HIV, TB/HIV co-infection, in advanced liver disease, children less than 5 years of age, and for key populations like female sex workers, MSM, transgender and intravenous drug users. With the current criteria for starting ART, HIV related mortality is expected to be reduced by $39 \%$.

\section{Targets needed to be achieved to end the AIDS"} epidemic by 2030

\section{- Ninety percent reduction in new adult HIV infections, including among key populations. \\ - Zero new infections among children. \\ - Ninety percent reduction in stigma and discrimination faced by people living with HIV and key populations.}

Ninety percent reduction in AIDS-related deaths.In order to achieve zero deaths related to HIV, we need to scale up our diagnostic and treatment services. We need better drugs with fewer side effects and improved service delivery models. An effective vaccine against HIV and a cure are the ultimate goals.

\section{What are the challenges?}

Presence of a large youth population, internal and external migration, clandestine but flourishing sex industry, low level of condom use and concurrent sexual relationship among key populations are some of the main challenges faced by the National Programme.

\section{How to overcome these challenges?}

To overcome these challenges and to achieve era free of HIV, we need to prioritize the targeted intervention to the key populations such as point of care testing. Raising public awareness about HIV through electronic and print media can play a major role towards achieving an era free of HIV. Unfortunately at present the media does not seems to identify this as an important area. Compared to the time allocated for areas like cookery, beauty culture, astrology and other medical discussions time allocated to HIV prevention is negligible. It is very costly to advertise in electronic/print media at present. Compared to other countries in the region transfusion acquired HIV infections are not reported from Sri 
Lanka recently. We should commend the National

Blood Transfusion Service and encourage them to continue their good work. Though HIV testing is available in all STD clinics people need to be encouraged to get tested without stigma and discrimination. To eliminate MTCT of HIV all antenatal mothers should be tested for HIV as a part of routine antenatal package. This can be easily achieved because nearly all mothers receive antenatal care in Sri Lanka. However, HIV testing should be extended to the private sector as some mothers receive antenatal care only at private sector. In addition, there should be a proper link between private sector and government STD clinics. 